\title{
Rhumatologie, la multitude des options
}

$>$ Le nombre d'anticorps (Ac) thérapeutiques disponibles en rhumatologie ne cesse de croître et concerne aussi bien les rhumatismes inflammatoires chroniques que les connectivites, les vascularites et, dans une moindre mesure, les pathologies osseuses et l'arthrose. Le choix d'un biomédicament repose aujourd'hui beaucoup sur les spécificités du médicament et des caractéristiques du patient. II n'existe pas encore de véritables biomarqueurs prédictifs de réponse pour une médecine plus personnalisée. Le suivi sérique des Ac thérapeutiques et le dosage des anticorps anti-médicaments représentent un espoir pour adapter au mieux la posologie du médicament et décider d'un changement de traitement en fonction de l'activité de la maladie inflammatoire chronique.<

Une meilleure compréhension des mécanismes contrôlant l'immunité et l'inflammation a permis d'identifier de nombreuses cibles thérapeutiques, telles que les cytokines, les protéines de surface cellulaire ou les voies de signalisation intracellulaires [1]. À partir du concept simple qu'un anticorps pouvait neutraliser une cible spécifique, associé aux progrès de la biologie moléculaire qui ont permis d'envisager la synthèse et la modification structurale d'anticorps monoclonaux (AcM), une nouvelle classe de médicament a ainsi vu le jour : les biomédicaments. Cette nouvelle classe thérapeutique représente l'un des progrès majeurs des $x x^{e}$ et $\mathrm{XXI}^{\mathrm{e}}$ siècles, particulièrement en rhumatologie. Depuis 20 ans, le nombre d'anticorps thérapeutiques disponibles en rhumatologie n'a cessé de croître et concerne aussi bien les rhumatismes inflammatoires chroniques que les connectivites, les vascularites et, dans une moindre mesure, les pathologies osseuses et l'arthrose.

\section{Anticorps thérapeutiques en rhumatologie}

Il existe actuellement deux principaux modes d'action des anticorps thérapeutiques utilisés en rhuma-
Jacques Morel ${ }^{1}$, Denis Mulleman ${ }^{2}$

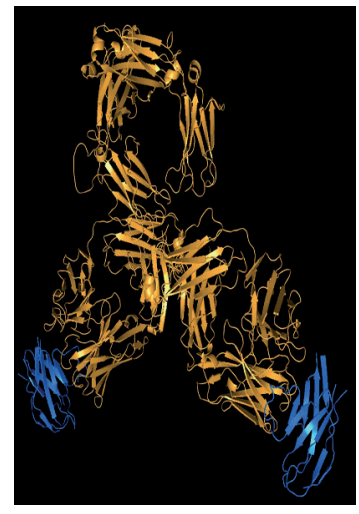

${ }^{1}$ Département de rhumatologie, Université et CHU de Montpellier ; UMR 5535, Institut de génétique moléculaire de Montpellier (IGMM), Montpellier, France. ${ }^{2}$ Université de Tours, EA 7501, Groupe insuffisance cardiaque et cardiomyopathie (GICC), CHRU de Tours, Service de rhumatologie, Tours, France. j-morel@chu-montpellier.fr

tologie. Le premier consiste à neutraliser une cytokine impliquée dans l'immunité ou un facteur de croissance (Figure l). Le premiers anticorps thérapeutique efficace pour traiter les rhumatismes inflammatoires a été un anticorps dirigé contre le TNF- $\alpha$ (tumor necrosis factor $\alpha$ ) [2]. D'autres cytokines pro-inflammatoires ont été ensuite ciblées comme l'interleukine (IL)-1 $\beta$ avec le canakinumab, ou I'IL-6. Des anticorps dirigés contre leur récepteur, comme le tocilizumab ou le sarilumab, qui reconnaissent le récepteur de l'IL-6, ont également été développés avec succès [3]. D'autres cytokines plus spécifiquement impliquées dans I'action des lymphocytes représentent également des cibles d'intérêt telles que le $B$ lymphocyte stimulator (BLyS). Ainsi, un anticorps anti-BLyS (aussi appelé BAFF), le bélimumab, bloque l'activation des lymphocytes B auto-réactifs [4]. Pour bloquer la voie des lymphocytes Th17, dont l'implication dans les rhumatismes inflammatoires a été largement démontrée, il existe plusieurs anticorps [5], dont certains neutralisent directement une ou plusieurs isoformes de l'IL-17, comme le sécukinumab, l'ixékizumab ou le bisékizumab. D'autres agissent plus en amont, en bloquant un des récepteurs de I'IL-23 qui active la sécrétion d'IL-17 par les lymphocytes Th17. L'ustékinumab agit ainsi sur la sous-unité $p 40$ du récepteur, tandis que le guselkumab et le risankizumab agissent sur sa sous-unité p19. Le deuxième mode d'action consiste à cibler une molécule membranaire spécifique d'une cellule qui peut soit réguler son activation, soit induire sa mort. Le rituximab (RTX) est ainsi un anticorps dirigé contre la molécule de surface CD20 qui est surtout exprimée sur les lymphocytes $B$ après le stade pré-B et avant leur différenciation en plasmocyte [6]. Le RTX induit une déplétion lymphocytaire $B C D 20^{+}$prolongée sur plusieurs mois qui explique une efficacité durable et un retraitement par RTX ne s'envisage pas avant 6 mois suivant le premier cycle de traitement. 


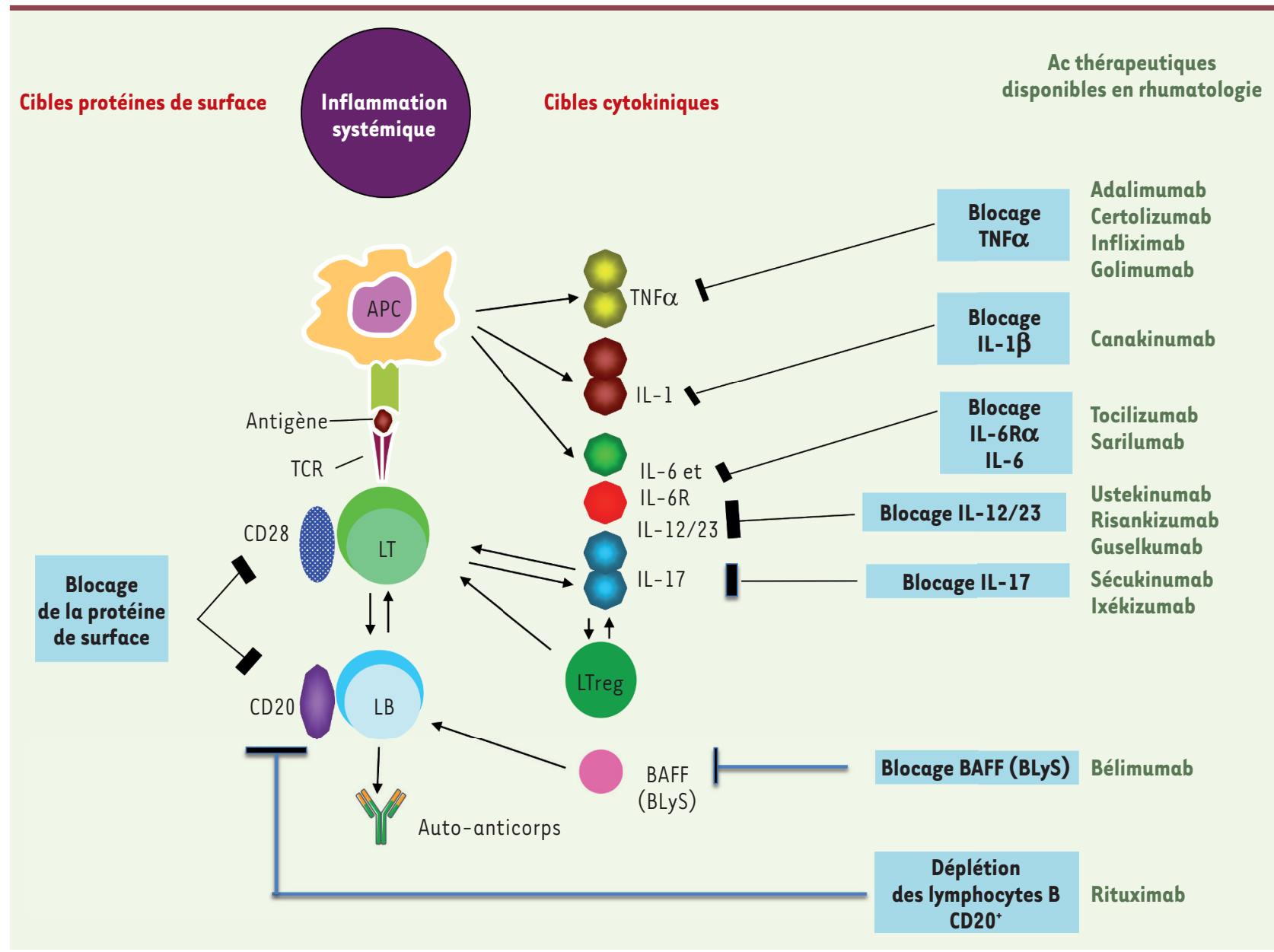

Figure 1. Anticorps thérapeutiques disponibles en rhumatologie dans les maladies inflammatoires à médiation immune. En rouge : les cibles (inhibition de l'action d'une cytokine cible ou d'un récepteur membranaire cible ou cytotoxicité [CD20]. En vert : les anticorps thérapeutiques (DCI).

\section{Les anticorps thérapeutiques dans les rhumatismes inflammatoires chroniques}

L'arrivée des biomédicaments a considérablement modifié la prise en charge des rhumatismes inflammatoires chroniques comme la polyarthrite rhumatoïde (PR) et les spondyloarthrites (SpA axiales; rhumatisme psoriasique) avec, aujourd'hui, des objectifs thérapeutiques plus ambitieux, tels que la rémission complète du rhumatisme inflammatoire et l'épargne des traitements anti-inflammatoires stéroïdiens et non stéroïdiens.

Le premier anticorps thérapeutique à avoir obtenu une autorisation en rhumatologie a été l'infliximab qui est un anticorps dirigé contre le TNF- $\alpha$ [7]. Du fait de leur grande efficacité sur les douleurs et les gonflements des articulations, I'inflammation biologique et la destruction articulaire observée dans la PR, d'autres AcM anti-TNF- $\alpha$ ont été développés: adalimumab, certolizumab pegol, golimumab [8]. Cette efficacité démontrée d'abord dans la PR sera ensuite élargie à d'autres rhumatismes inflammatoires chroniques, comme la spondylarthrite ankylosante (SA), les spondyloarthrites non radiographiques, le rhumatisme psoriasique, ou encore les arthrites juvéniles $[9,10]$. Ce succès médical et commercial conduira au développement de nombreux autres AcM. Ainsi, un anticorps monoclonal humanisé dirigé contre la chaîne alpha du récepteur de l'IL-6, le tocilizumab (TCZ), démontrera d'abord son efficacité dans la PR [11]. Ce biomédicament va bloquer à la fois l'activation directe de la cellule par l'IL-6 et l'activation indirecte par trans-signalisation. Dans la PR, le TCZ a montré une efficacité au moins comparable à celle des anti-TNF- $\alpha$. Depuis le TCZ, d'autres AcM dirigés contre le récepteur de l'IL-6 ont été développés, comme le sarilumab qui vient d'obtenir une autorisation de mise sur le marché (AMM) dans la PR [12]. Après les anticorps anti-cytokines, la modulation des lymphocytes a été rendue possible grâce à la mise à disposition du rituximab, un AcM initialement développé en hémato-oncologie, puis testé avec efficacité dans la PR. Cet AcM chimérique entraîne une déplétion périphérique lymphocytaire $B$ 
qui s'accompagne souvent d'une diminution des auto-anticorps, facteur rhumatoïde et anticorps anti-protéines citrullinées. Ces anticorps thérapeutiques, une fois testés dans les rhumatismes inflammatoires chroniques (RIC) sont ensuite souvent évalués dans d'autres maladies auto-immunes ou inflammatoires. Ainsi, le rituximab est aujourd'hui proposé dans le traitement des vascularites à ANCA (auto-anticorps anti-cytoplasmiques de neutrophiles) et le tocilizumab dans l'artérite à cellules géantes $[13,14]$.

Les anticorps thérapeutiques permettent également d'apporter des informations importantes sur l'implication des cytokines dans la physiopathologie des RIC [15]. En effet, si les anti-TNF- $\alpha$ avaient démontré une efficacité aussi bien dans la PR que dans les SpA, la neutralisation de I'IL-6 s'est avérée peu efficace dans les SpA [16]. Inversement, des anticorps ciblant I'IL-17 se sont montrés peu efficaces dans la PR alors que leur efficacité a été démontrée dans les SpA axiales et périphériques [17]. Deux anticorps anti-IL-17, le sécukinumab et l'ixékizumab, ont en effet obtenu une AMM dans la spondylarthrite ankylosante et le rhumatisme psoriasique [18]. Cette implication différente des cytokines IL-17 et IL-6 dans la PR et les SpA n'est pas encore bien expliquée. Il est possible que ces cytokines interviennent à des périodes différentes du développement du rhumatisme inflammatoire. Une autre explication serait que I'IL-6 et I'IL-17 jouent un rôle distinct dans le contrôle de la différenciation des lymphocytes T régulateurs. Cibler la voie Th17 en neutralisant le récepteur de l'IL-23 avec l'ustékinumab, le guselkumab et le risankizumab apparaît particulièrement efficace dans le rhumatisme psoriasique qui s'exprime principalement par des manifestations articulaires périphériques, même si l'atteinte axiale peut être observée [15]. Par contre, cibler le récepteur de I'IL-23 est inefficace dans l'atteinte axiale des SpA. En effet, les essais thérapeutiques avec l'ustékinumab, tout comme avec le risankizumab n'ont pas permis de démontrer une efficacité dans la spondylarthrite ankylosante $[19,20]$. Ces différences d'efficacité entre le ciblage direct de l'IL-17 et le ciblage de l'IL-23 sur l'atteinte axiale restent actuellement une énigme. Des études translationnelles pourraient permettre à l'avenir de préciser le mécanisme physiopathologique de chacun de ces deux rhumatismes.

\section{Médecine personnalisée et prédictive, suivi thérapeutique pharmacologique}

Le principe du meilleur choix thérapeutique pour un patient donné (personalized medicine) s'applique aux biomédicaments utilisés dans les rhumatismes inflammatoires chroniques. La recherche de marqueurs cliniques ou biologiques prédictifs de réponse à un traitement représente également un enjeu de taille. Si certaines caractéristiques du patient ou de sa pathologie sont associées à la réponse clinique à telle ou telle molécule, il n'existe pas pour le moment de classification (patient stratification) pour définir a priori quels patients atteints de la même maladie inflammatoire chronique seraient plus répondeurs à un traitement plutôt qu'à un autre. Ces dernières années, le suivi thérapeutique pharmacologique (therapeutic drug monitoring, ou TDM) a connu un intérêt auprès des sociétés savantes et des industriels pour essayer d'adapter au mieux la dose d'anticorps thérapeutique à l'échelon individuel. C'est ainsi que la Société savante européenne EULAR (european league against rheumatism) a créé en 2018 un groupe de travail sur ce sujet [21]. Le principe du TDM est d'ajuster la posologie d'une molécule selon les caractéristiques du patient, comme par exemple l'activité de la maladie, afin d'obtenir la concentration sérique de médicament souhaitée et ainsi atteindre la réponse clinique. Cela présuppose qu'il existe une relation entre la dose et la concentration du biomédicament (pharmacocinétique) et entre la concentration et la réponse thérapeutique (relation pharmacocinétique-pharmacodynamique). II existe trois principaux arguments en faveur du TDM des biomédicaments anti-TNF- $\alpha$ chez les patients atteints de rhumatismes inflammatoires chroniques [22]. Premièrement, l'existence d'une variabilité pharmacocinétique. En effet les concentrations d'AcM anti-TNF- $\alpha$ varient considérablement entre les patients, certains présentant des concentrations indétectables, alors que d'autres sont manifestement surexposés [23]. Deuxièmement, l'existence d'une relation concentration-réponse. Celle-ci a largement été rapportée avec l'adalimumab dans la PR. Les patients ayant obtenu une bonne réponse au traitement avaient des concentrations plus élevées que les patients avec une réponse modérée ou ne présentant aucune réponse [24], suggérant que la concentration est un des facteurs de réponse aux anticorps anti-TNF- $\alpha$. Troisièmement, un index thérapeutique étroit. En effet, une faible exposition lors de l'initiation ou après une vacance thérapeutique est associée à un sur-risque de développer des anticorps anti-médicaments (ADA pour anti-drug antibodies), comme cela a été démontré avec l'infliximab dans la PR et dans la spondyloarthrite [25, 26]. À l'opposé, de fortes concentrations résiduelles d'infliximab sont associées à une multiplication du risque de survenue d'infections d'un facteur 2 à 3 dans la SpA [27] et d'un facteur 1,5 dans la PR [28].

L'objectif principal du TDM des anticorps thérapeutiques dans les rhumatismes inflammatoires est d'améliorer la réponse clinique et la tolérance. Le TDM pourrait également améliorer le rapport coût-efficacité [29]. Récemment, chez des patients traités par adalimumab et ayant une concentration résiduelle supérieure à $8 \mu \mathrm{g} / \mathrm{mL}$, il a été démontré qu'espacer les injections toutes les trois semaines au lieu de deux n'induisait pas de perte d'efficacité, suggérant la possibilité d'une économie [30]. En utilisant un modèle pharmacocinétique-pharmacodynamique pour décrire la relation concentration-réponse, il a été possible de définir la concentration cible nécessaire pour obtenir une faible 


\begin{tabular}{|c|c|c|c|}
\hline \multicolumn{2}{|c|}{ Exposition et immunogénicité } & \multicolumn{2}{|c|}{ Réponse clinique } \\
\hline Concentration résiduelle & ADA & Répondeur & Non-répondeur \\
\hline Au-dessus de l'intervalle cible & NA & Diminuer la posologie & Switch pour une autre classe \\
\hline Dans l'intervalle cible & NA & Même posologie & Switch pour une autre classe \\
\hline \multirow[t]{2}{*}{ En dessous de l'intervalle cible } & Indétectable ${ }^{a}$ & $\begin{array}{l}\text { Discuter l'augmentation de la posologie } \\
\text { ou décider la même posologie ou l'arrêt } \\
\text { du traitement }{ }^{b}\end{array}$ & Augmentation de la posologie \\
\hline & Détectable $^{a}$ & Discuter l'arrêt du traitement & $\begin{array}{l}\text { Discuter une augmentation } \\
\text { de la posologie ou switch }\end{array}$ \\
\hline \multirow[t]{2}{*}{ Indétectable } & Indétectable ${ }^{d}$ & $\begin{array}{l}\text { Discuter l'augmentation de la posologie ou } \\
\text { l'arrêt du traitement }{ }^{\text {b }}\end{array}$ & Augmentation de la posologie \\
\hline & Détectable $^{d}$ & Discuter l'arrêt du traitement & Switch $^{c}$ \\
\hline $\begin{array}{l}\text { L'algorithme aide à la décision } \\
\text { de la détection d'ADA }\end{array}$ & réponse clinic & mesure biologique de la concentration de I & njecté et, si nécessaire, \\
\hline
\end{tabular}

Tableau I. Algorithme décisionnel pour le TDM des biomédicaments dans les maladies inflammatoires (d'après [33]). a Possibilité d'utiliser un test drug-tolerant pour la détection des ADA. 'La décision du clinicien peut varier selon les traitements disponibles et les indications. ${ }^{~} S w i t c h$ intraclasse ou bien vers une autre classe. "Utiliser un test "drug-sensitive" pour la détection des ADA. Toujours vérifier la bonne observance du traitement en cas d'auto-administration, particulièrement lorsque la concentration est en-dessus de l'intervalle cible. Un changement de posologie reste la décision du clinicien et nécessite une réévaluation de la réponse ainsi qu'une vérification de la concentration. ADA, antidrug antibodies ; NA, non-applicable ; TDM, therapeutic drug monitoring (suivi thérapeutique pharmacologique).

activité de la PR selon le score DAS281 (disease activity score 28) en prenant en compte l'activité de la maladie au moment de l'initiation du traitement. Si cette approche est prometteuse afin d'établir une posologie individualisée, ce résultat nécessite une validation prospective avant de pouvoir s'appliquer en pratique [31].

Certains centres utilisent déjà le suivi des concentrations d'anticorps thérapeutiques (pour l'essentiel des anticorps anti-TNF- $\alpha$ ) ainsi que la détection des ADA. Lorsqu'un biomédicament est utilisé pour un rhumatisme inflammatoire, les principales situations rencontrées sont la non-réponse, la perte de réponse, la rémission clinique de la maladie et la réaction clinique à l'injection, parfois grave. L'évaluation de l'activité de la maladie par les scores, tels que I'ASDAS 2 pour les SpA ou le DAS28 pour la PR d'une part, et la concentration de médicament et d'ADA d'autre part, sont des éléments qui pourraient aider le clinicien dans sa décision vis-à-vis de la poursuite du traitement et de l'adaptation de la dose ou de l'espacement entre les prises. La concentration résiduelle apporte une information sur l'exposition du patient au traitement ainsi qu'une probabilité de s'être immunisé contre l'anticorps thérapeutique. La détection des ADA est critique, particulièrement en cas de réaction à l'injection, et il est nécessaire d'avoir un test fiable pour distinguer les réactions liées à l'immunogénicité de l'anticorps de

${ }^{1}$ Le DAS28 tient compte de l'évaluation de l'activité de la PR, du nombre d'articulations douloureuses et gonflées sur 28 sites articulaires et de la vitesse de sédimentation.

${ }^{2}$ Ankylosing spondylitis disease activity score : http ://medicalcul.free.fr/asdas.html. celles qui sont liées à d'autres mécanismes. Pour éviter les faux négatifs, il faut disposer d'un test qui ne soit pas sensible à l'interférence avec le médicament (drug tolerant assays). Des algorithmes ont été proposés par plusieurs équipes pour adapter la dose de l'anticorps thérapeutique ou changer de traitement. Un exemple est fourni par celui proposé par le groupe MAGE (monitoring of monoclonal antibodies group in Europe) [32, 33] (Tableau I).

\section{Conclusion}

Pour conclure, les anticorps thérapeutiques utilisés en rhumatologie sont très majoritairement des molécules dirigées contre des cytokines. Leur spécificité de cibles a permis d'améliorer la connaissance des maladies rhumatologiques inflammatoires (cytokine-based disease classification). Si cette multitude d'options thérapeutiques a considérablement élargi l'éventail thérapeutique dans les maladies inflammatoires et auto-immunes, de nombreuses questions restent en suspens comme l'identification de biomarqueurs prédictifs de réponse et de tolérance à ces traitements. L'optimisation de l'efficacité et de la tolérance des biomédicaments par le monitoring de leurs concentrations sériques reste une question d'actualité. Avec les 
progrès de la connaissance de la relation dose-concentration-réponse des anticorps thérapeutiques, en particulier des anticorps anti-TNF- $\alpha$, le suivi thérapeutique de ces biomédicaments pourrait devenir la pratique courante dans le futur pour les malades atteints de rhumatismes inflammatoires chroniques. Plusieurs études sont cependant encore nécessaires avant une utilisation en routine de ces tests. $\diamond$

\section{SUMMARY}

\section{Rheumatology, the multitude of options}

The number of therapeutic antibodies available in rheumatology increases every year, mainly indicated in chronic inflammatory rheumatisms and other immune-mediated inflammatory diseases. The choice between all these monoclonal antibodies depends on their specificities, patients and diseases characteristics. Until now, there is no reliable biomarker, predictive of response for specialized medicine purpose. Today, therapeutic drug monitoring and anti-drug antibodies testing can help to better adapt the dose of the drug and to help in the decision to switch to another biological drug according to the activity of the inflammatory disease. $\diamond$

\section{LIENS D'INTÉRÊT}

JM déclare n'avoir aucun lien d'intérêt concernant les données publiées dans cet article. Au cours des trois dernières années, DM a donné des conférences au titre de son université pour Grifols, Novartis et Pfizer. Il a été invité à participer à un congrès international par Janssen-Cilag et son université a reçu des financements du Lions Club Tours Val-de-France.

\section{RÉFÉRENCES}

1. Mclnnes IB, Schett G. The pathogenesis of rheumatoid arthritis. N Engl J Med 2011 ; 365 : 2205-19.

2. Elliott MJ, Maini RN, Feldmann M, et al. Randomised double-blind comparison of chimeric monoclonal antibody to tumour necrosis factor alpha (cA2) versus placebo in rheumatoid arthritis. Lancet $1994 ; 344: 1105-10$.

3. Mclnnes IB, Schett G. Pathogenetic insights from the treatment of rheumatoid arthritis. Lancet $2017 ; 389: 2328-37$.

4. Stohl W. Future prospects in biologic therapy for systemic lupus erythematosus. Nat Rev Rheumatol $2013 ; 9: 705-20$.

5. Mease PJ. Inhibition of interleukin-17, interleukin-23 and the TH17 cell pathway in the treatment of psoriatic arthritis and psoriasis. Curr Opin Rheumatol $2015 ; 27: 127-33$.

6. Edwards JC, Cambridge G. B-cell targeting in rheumatoid arthritis and other autoimmune diseases. Nat Rev Immunol $2006 ; 6$ : 394-403.

7. Maini RN, Breedveld FC, Kalden JR, et al. Therapeutic efficacy of multiple intravenous infusions of anti-tumor necrosis factor alpha monoclonal antibody combined with low-dose weekly methotrexate in rheumatoid arthritis. Arthritis Rheum 1998; 41 : 1552-63.

8. Nam JL, Takase-Minegishi K, Ramiro S, et al. Efficacy of biological disease-modifying antirheumatic drugs : a systematic literature review informing the 2016 update of the EULAR recommendations for the management of rheumatoid arthritis. Ann Rheum Dis $2017 ; 76: 1113-36$.

9. Braun J, Kiltz U, Heldmann F, Baraliakos X. Emerging drugs for the treatment of axial and peripheral spondyloarthritis. Exp Opin Emerg Drugs $2015 ; 20: 1-14$.

10. Otten MH, Anink J, Spronk S, van Suijlekom-Smit LW. Efficacy of biological agents in juvenile idiopathic arthritis : a systematic review using indirect comparisons. Ann Rheum Dis 2013; 72 : 1806-12.

11. Singh JA, Beg S, Lopez-Olivo MA. Tocilizumab for rheumatoid arthritis. Cochrane Database Syst Rev 2010 : CD008331.

12. Scott LJ. Sarilumab : first global approval. Drugs $2017 ; 77: 705-12$.

13. Stone JH, Klearman M, Collinson N. Trial of Tocilizumab in giant-cell arteritis. N Engl J Med 2017 ; 377 : 1494-5.

14. Stone JH, Merkel PA, Spiera R, et al. Rituximab versus cyclophosphamide for ANCA-associated vasculitis. N Engl J Med $2010 ; 363: 221-32$.

15. Baker KF, Isaacs JD. Novel therapies for immune-mediated inflammatory diseases : what can we learn from their use in rheumatoid arthritis, spondyloarthritis, systemic lupus erythematosus, psoriasis, Crohn's disease and ulcerative colitis? Ann Rheum Dis 2018 ; 77 : 175-87.

16. Van den Bosch F, Deodhar A. Treatment of spondyloarthritis beyond TNF-alpha blockade. Best Practice Res Clin Rheumatol $2014 ; 28: 819-27$.
17. Kunwar S, Dahal K, Sharma S. Anti-IL-17 therapy in treatment of rheumatoid arthritis: a systematic literature review and meta-analysis of randomized controlled trials. Rheumatol Int 2016; 36 : 1065-75.

18. Sieper J, Poddubnyy D. New evidence on the management of spondyloarthritis. Nat Rev Rheumato $2016 ; 12: 282-95$.

19. Baeten D, Ostergaard M, Wei JC, et al. Risankizumab, an IL-23 inhibitor, for ankylosing spondylitis : results of a randomised, double-blind, placebocontrolled, proof-of-concept, dose-finding phase 2 study. Ann Rheum Dis $2018 ; 77: 1295-302$.

20. Poddubnyy D, Hermann KG, Callhoff J, et al. Ustekinumab for the treatment of patients with active ankylosing spondylitis: results of a 28-week, prospective, open-label, proof-of-concept study (TOPAS). Ann Rheum Dis $2014 ; 73: 817-23$.

21. EULAR therapeutic drug monitoring study group. https ://wwweularorg/ clinical_affairs_study_groupscfm

22. Mulleman D, Balsa A. Adalimumab concentration-based tapering strategy: as good as the recommended dosage. Ann Rheum Dis 2018 ; 77 : 473-5.

23. St Clair EW, Wagner CL, Fasanmade AA, et al. The relationship of serum infliximab concentrations to clinical improvement in rheumatoid arthritis: results from ATTRACT, a multicenter, randomized, double-blind, placebocontrolled trial. Arthritis Rheum $2002 ; 46: 1451-9$.

24. Bartelds GM, Wijbrandts CA, Nurmohamed MT, et al. Clinical response to adalimumab: relationship to anti-adalimumab antibodies and serum adalimumab concentrations in rheumatoid arthritis. Ann Rheum Dis 2007 ; $66: 921-6$.

25. Bendtzen K, Geborek P, Svenson M, et al. Individualized monitoring of drug bioavailability and immunogenicity in rheumatoid arthritis patients treated with the tumor necrosis factor alpha inhibitor infliximab. Arthritis Rheum $2006 ; 54: 3782-9$.

26. Ducourau E, Mulleman D, Paintaud G, et al. Antibodies toward infliximab are associated with low infliximab concentration at treatment initiation and poor infliximab maintenance in rheumatic diseases. Arthritis Res Ther 2011 ; $13:$ R105.

27. Bejan-Angoulvant T, Ternant D, Daoued F, et al. Brief report : relationship between serum infliximab concentrations and risk of infections in patients treated for spondyloarthritis. Arthritis Rheumatol 2017 ; 69: 108-13.

28. Jani M, Dixon WG, Lunt $M$, et al. The association of biologic drug-levels with infection risk : results from the british society for rheumatology biologics register for rheumatoid arthritis. EULAR annual meeting. Ann Rheum Dis 2018: Al63.

29. Krieckaert CL, Nair SC, Nurmohamed MT, et al. Personalised treatment using serum drug levels of adalimumab in patients with rheumatoid arthritis: an evaluation of costs and effects. Ann Rheum Dis 2015 ; 74 : 361-8.

30. L'Ami MJ, Krieckaert CL, Nurmohamed MT, et al. Successful reduction of overexposure in patients with rheumatoid arthritis with high serum adalimumab concentrations: an open-label, non-inferiority, randomised clinical trial. Ann Rheum Dis $2018 ; 77: 484-7$.

31. Ducourau $\varepsilon$, Ternant D, Lequerre T, et al. Towards an individualised target concentration of adalimumab in rheumatoid arthritis. Ann Rheum Dis 2014 ; $73: 1428-9$.

32. Monitoring of monoclonal antibodies group in Europe (MAGE) for inflammatory diseases. wwwlestudium-iascom/content/monitoringmonoclonal-antibodies-group-europe-mage-inflammatory-diseases

33. Dreesen $\varepsilon$, Bossuyt P, Mulleman D, et al. Practical recommendations for the use of therapeutic drug monitoring of biopharmaceuticals in inflammatory diseases. Clin Pharmacol 2017 ; 9 : 1-11.

TIRÉS À PART

J. Morel

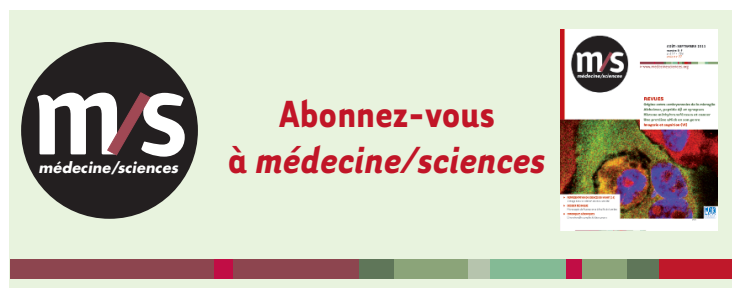

Bulletin d'abonnement page 1230 dans ce numéro de $\mathrm{m} / \mathrm{s}$ 